\title{
Multicoloured Hamilton cycles in random graphs; an anti-Ramsey threshold.
}

\author{
Colin Cooper \\ School of Mathematical Sciences, \\ University of North London, \\ London N7 8DB, U.K. *
}

\author{
Alan Frieze \\ Department of Mathematics, \\ Carnegie Mellon University, \\ Pittsburgh PA15213, U.S.A. ${ }^{\dagger}$
}

Submitted: August 25, 1995; Accepted October 1, 1995

\begin{abstract}
Let the edges of a graph $G$ be coloured so that no colour is used more than $k$ times. We refer to this as a $k$-bounded colouring. We say that a subset of the edges of $G$ is multicoloured if each edge is of a different colour. We say that the colouring is $\mathcal{H}$-good, if a multicoloured Hamilton cycle exists i.e., one with a multicoloured edge-set.

Let $\mathcal{A R}_{k}=\{G$ : every $k$-bounded colouring of $G$ is $\mathcal{H}$-good $\}$. We establish the threshold for the random graph $G_{n, m}$ to be in $\mathcal{A R}_{k}$.
\end{abstract}

\footnotetext{
${ }^{*}$ Research carried out whilst visiting Carnegie Mellon University

†'Supported by NSF grant CCR-9225008
} 
THE ELECTRONiC JOURNAL OF COMBinAtorics 2 (1995), \#R19

\section{Introduction}

As usual, let $G_{n, m}$ be the random graph with vertex set $V=[n]$ and $m$ random edges. Let $m=n\left(\log n+\log \log n+c_{n}\right) / 2$. Komlós and Szemerédi [14] proved that if $\lambda=e^{-c}$ then

$$
\lim _{n \rightarrow \infty} \operatorname{Pr}\left(G_{n, m} \text { is Hamiltonian }\right)= \begin{cases}0 & c_{n} \rightarrow-\infty \\ e^{-\lambda} & c_{n} \rightarrow c \\ 1 & c_{n} \rightarrow \infty\end{cases}
$$

which is $\lim _{n \rightarrow \infty} \operatorname{Pr}\left(\delta\left(G_{n, m}\right) \geq 2\right)$, where $\delta$ refers to minimum degree.

This result has been generalised in a number of directions. Bollobás [3] proved a hitting time version (see also Ajtai, Komlós and Szemerédi [1]); Bollobás, Fenner and Frieze [6] proved an algorithmic version; Bollobás and Frieze [5] found the threshold for k/2 edge disjoint Hamilton cycles; Bollobás, Fenner and Frieze [7] found a threshold when there is a minimum degree condition; Cooper and Frieze [9], Łuczak [15] and Cooper [8] discussed pancyclic versions; Cooper and Frieze [10] estimated the number of distinct Hamilton cycles at the threshold.

In quite unrelated work various researchers have considered the following problem: Let the edges of a graph $G$ be coloured so that no colour is used more than $k$ times. We refer to this as a $k$-bounded colouring. We say that a subset of the edges of $G$ is multicoloured if each edge is of a different colour. We say that the colouring is $\mathcal{H}$-good, if a multicoloured Hamilton cycle exists i.e., one with a multicoloured edge-set. A sequence of papers considered the case where $G=K_{n}$ and asked for the maximum growth rate of $k$ so that every $k$-bounded colouring is $\mathcal{H}$-good. Hahn and Thomassen [13] showed that $k$ could grow as fast as $n^{1 / 3}$ and conjectured that the growth rate of $k$ could in fact be linear. In unpublished work Rödl and Winkler [18] in 1984 improved this to $n^{1 / 2}$. Frieze and Reed [12] showed that there is an absolute constant $A$ such that if $n$ is sufficiently large and $k$ is at most $\lceil n /(A \ln n)\rceil$ then any $k$-bounded colouring is $\mathcal{H}$-good. Finally, Albert, Frieze and Reed [2] show that $k$ can grow as fast as $c n, c<1 / 32$.

The aim of this paper is to address a problem related to both areas of activity. Let $\mathcal{A R}_{k}=\{G$ : 
every $k$-bounded colouring of $G$ is $\mathcal{H}$-good $\}$. We establish the threshold for the random graph $G_{n, m}$ to be in $\mathcal{A} \mathcal{R}_{k}$.

Theorem 1 If $m=n\left(\log n+(2 k-1) \log \log n+c_{n}\right) / 2$ and $\lambda=e^{-c}$, then

$$
\begin{aligned}
\lim _{n \rightarrow \infty} \operatorname{Pr}\left(G_{n, m} \in \mathcal{A} \mathcal{R}_{k}\right) & = \begin{cases}0 & c_{n} \rightarrow-\infty \\
\sum_{i=0}^{k-1} \frac{e^{-\lambda} \lambda^{i}}{i !} & c_{n} \rightarrow c \\
1 & c_{n} \rightarrow \infty\end{cases} \\
& =\lim _{n \rightarrow \infty} \operatorname{Pr}\left(G_{n, m} \in \mathcal{B}_{k}\right),
\end{aligned}
$$

where $\mathcal{B}_{k}=\{G: G$ has at most $k-1$ vertices of degree less than $2 k\}$.

Note that the case $k=1$ generalises the original theorem of Komlòs and Szemerèdi. We use $\mathcal{A R}_{k}$ to denote the anti-Ramsey nature of the result and remark that there is now a growing literature on the subject of the Ramsey properties of random graphs, see for example the paper of Rödl and Ruciński [17].

\section{Outline of the proof of Theorem 1}

We will prove the result for the independent model $G_{n, p}$ where $p=2 \mathrm{~m} / n$ and rely on the monotonicity of property $\mathcal{A R}_{k}$ to give the theorem as stated, see Bollobás [4] and Łuczak [16]. With a little more work, one could obtain the result that the hitting times for properties $\mathcal{A R}_{k}$ and $\mathcal{B}_{k}$ in the graph process are coincidental $\mathbf{w h} \mathbf{p}^{1}$.

We will follow the basic idea of [12] that, given a $k$-bounded colouring we will choose a multicoloured set of edges $E_{1} \cup E_{2}$ and show that whp $H=\left(V=[n], E_{1} \cup E_{2}\right)$ contains a Hamilton cycle. $E_{1}$ is chosen randomly, pruned of multiple colours and colours that occur on edges incident with vertices of low degree. $E_{2}$ is chosen carefully so as to ensure that vertices of low degree get at least 2 incident

\footnotetext{
${ }^{1}$ with high probability i.e. probability 1 -o(1) as $n \rightarrow \infty$
} 
THE ELECTRONiC JOURNAL OF COMBinAtorics 2 (1995), \#R19

edges and vertices of large degree get a substantial number of incident edges. $H$ is multicoloured by construction. We then use the approach of Ajtai, Komlós and Szemerédi [1] to show that $H$ is Hamiltonian whp.

\section{$3 \quad$ Required graph properties}

We say a vertex $v$ of $G=G_{n, p}$ is small if its degree $d(v)$ satisfies $d(v)<\log n / 10$ and large otherwise. Denote the set of small vertices by SMALL and the remaining vertices by LARGE. For $S \subseteq V$ we let

$$
N_{G}(S)=N(S)=\{w \notin S: \exists v \in S \text { such that }\{v, w\} \text { is an edge of } G\} \text {. }
$$

We now give a rather long list of properties. We claim

Lemma 1 If $p=(\log n+(2 k-1) \log \log n+c) / n$ then $G_{n, p}$ has properties $P 1-P 9$ below whp and property $P 10$ with probability equal to the RHS of (1).

P1 $\mid$ SMALL $\mid \leq n^{1 / 3}$.

P2 SMALL contains no edges.

P3 No $v \in V$ is within distance 2 of more than one small vertex.

P4 $S \subseteq$ LARGE, $|S| \leq n / \log n$ implies that $|N(S)| \geq|S| \log n / 20$.

P5 $T \subseteq V,|T| \leq n /(\log n)^{2}$ implies $T$ contains at most $3|T|$ edges.

P6 $A, B \subseteq V, A \cap B=\emptyset,|A|,|B| \geq 15 n \log \log n / \log n$ implies $G$ contains at least $|A||B| \log n / 2 n$ edges joining $A$ and $B$.

P7 $A, B \subseteq V, A \cap B=\emptyset,|A| \leq|B| \leq 2|A|$ and $|B| \leq D n \log \log n / \log n(D \geq 1)$ implies that there are at most $10 D|A| \log \log n$ edges joining $A$ and $B$. 
THE ELECTRONiC JOURNAL OF COMBinAtorics 2 (1995), \#R19

P8 If $|A| \leq D n \log \log n / \log n(D \geq 1)$ then $A$ contains at most $10 D|A| \log \log n$ edges.

P9 $G$ has minimum degree at least $2 k-1$.

P10 $G$ has at most $k-1$ vertices of degree $2 k-1$.

The proof that $G_{n, p}$ has properties P1-P4 whp can be carried out as in [6]. Erdős and Rényi [11] contains our claim about P9, P10. The remaining claims are simple first moment calculations and are placed in the appendix.

\section{A simple necessary condition}

We now show the relevance of P9, P10. Suppose a graph $G$ has $k$ vertices $v_{1}, v_{2}, \ldots, v_{k}$ of degree $2 k-1$ or less and these vertices form an independent set. (The latter condition is not really necessary.) We can use colour $2 i-1$ at most $k$ times and colour $2 i$ at most $k-1$ times to colour the edges incident with $v_{i}, 1 \leq i \leq k-1$. Now use colours $2,4,6, \ldots, 2 k-2$ at most once and colour $2 k-1$ at most $k$ times to colour the edges inicident with $v_{k}$. No matter how we colour the other edges of $G$ there is no multicoloured Hamilton cycle. Any such cycle would have to use colours $1,2, \ldots, 2 k-2$ for its edges incident with $v_{1}, v_{2}, \ldots, v_{k-1}$ and then there is only one colour left for the edges incident with vertex $v_{k}$.

Let $\mathcal{N}_{k}$ denote the set of graphs satisfying P1-P10. It follows from Lemma 1 and the above that we can complete the proof of Theorem 1 by proving

$$
\mathcal{N}_{k} \subseteq \mathcal{A} \mathcal{R}_{k}
$$


THE ELECTRONiC Journal of COMBinatorics 2 (1995), \#R19

\section{Binomial tails}

We make use of the following estimates of tails of the Binomial distribution several times in subsequent proofs.

Let $X$ be a random variable having a $\operatorname{Binomial~distribution~} \operatorname{Bin}(n, p)$ resulting from $n$ independent trials with probability $p$. If $\mu=n p$ then

$$
\begin{array}{ll}
\operatorname{Pr}(X \leq \alpha \mu) \leq\left(\frac{e}{\alpha}\right)^{\alpha \mu} e^{-\mu} & 0<\alpha \leq 1 \\
\operatorname{Pr}(X \geq \alpha \mu) \leq\left(\frac{e}{\alpha}\right)^{\alpha \mu} e^{-\mu} & 1 \leq \alpha .
\end{array}
$$

\section{Main Proof}

Assume from now on that we have a fixed graph $G=(V, E) \in \mathcal{N}_{k}$. We randomly select a multicoloured subgraph $H$ of $G, H=\left(V, E_{1} \cup E_{2}\right)$ and prove that it is Hamiltonian whp. From now on all probabilistic statements are with respect to the selection of the random set $E_{1} \cup E_{2}$ and not the choice of $G=G_{n, p}$.

\subsection{Construction of the multicoloured subgraph $H$}

The sets $E_{1}$ and $E_{2}$ are obtained as follows.

\subsubsection{Selection of $E_{1}$}

(i) Choose edges of the subgraph of $G$ induced by LARGE independently with probability $\epsilon / k, \epsilon=$ $e^{-200 k}$, to obtain $\widetilde{E}_{1}$. 
THE ELECTROniC Journal of COMBinatorics 2 (1995), \#R19

(ii) Remove from $\widetilde{E}_{1}$ all edges whose colour occurs more than once in $\widetilde{E}_{1}$ and also edges whose colour is the same as that of any edge incident with a small vertex.

Denote the edge set chosen by $E_{1}$, and denote by $E_{1}^{\star}$ the subset of edges of $E$ which have the same colour as that of an edge in $E_{1}$.

Lemma 2 For $v \in L A R G E$ let $d^{\prime}(v)$ denote the degree of $v$ in $\left(V, E \backslash E_{1}^{\star}\right)$. Then whp

$$
d^{\prime}(v)>\frac{9}{100 k} \log n
$$

for all $v \in L A R G E$.

Proof Suppose that large vertex $v$ has edges of $r=r(v)$ different colours $c_{1}, c_{2}, \ldots, c_{r}$ incident with it in $G$, where $d(v) / k \leq r \leq d(v)$. Let $X_{i}, 1 \leq i \leq r$ be an indicator for the event that $E_{1}$ contains an edge of colour $c_{i}$ which is incident with $v$. Let $k_{i}$ denote the number of times colour $c_{i}$ is used in $G$ and let $\ell_{i}$ denote the number of edges of colour $c_{i}$ which are incident with $v$. Then

$$
\begin{aligned}
\operatorname{Pr}\left(X_{i}=1\right) & \leq \ell_{i} \frac{\epsilon}{k}\left(1-\frac{\epsilon}{k}\right)^{k_{i}-1} \\
& \leq \epsilon .
\end{aligned}
$$

The random variables $X_{1}, X_{2}, \ldots, X_{r}$ are independent and so $X=X_{1}+X_{2}+\cdots+X_{r}$ is dominated by $\operatorname{Bin}(r, \epsilon)$. Thus, by (4),

$$
\begin{aligned}
\operatorname{Pr}\left(X \geq \frac{r}{10}\right) & \leq(10 e \epsilon)^{\frac{r}{10}} \\
& \leq(10 e \epsilon)^{\frac{\log n}{100 k}} \\
& \leq n^{-3 / 2}
\end{aligned}
$$

when $\epsilon=e^{-200 k}$. Hence whp,

$$
d^{\prime}(v)>\frac{9}{10} r \geq \frac{9}{100 k} \log n
$$


THE ELECTRONIC JOURNAL OF COMBINATORICS 2 (1995), \#R19

for every $v \in$ LARGE.

Assume then that

$$
d^{\prime}(v)>\frac{9}{100 k} \log n
$$

for $v \in$ LARGE.

\subsubsection{Selection of $E_{2}$}

We show we can choose a monochromatic subset $E_{2}$ of $E \backslash E_{1}^{*}$ in which

D1 The vertices of SMALL have degree at least 2,

D2 The vertices of LARGE have degree at least $\left\lfloor\frac{9}{200 k^{2}} \log n\right\rfloor$.

In order to select $E_{2}$, we first describe how to choose for each vertex $v \in V$, a subset $A_{v}$ of the edges of $E \backslash E_{1}^{\star}$ incident with $v$. These sets $A_{v}, v \in V$ are pairwise disjoint.

The vertices $v$ of SMALL are independent (P2) and we take $A_{v}$ to be the set of edges incident with $v$ if $d(v)=2 k-1$, and $A_{v}$ to be an $m k$ subset otherwise, where $m=\lfloor d(v) / k\rfloor$.

The subgraph $F$ of $E \backslash E_{1}^{\star}$ induced by LARGE, is of minimum degree greater than $(9 \log n) / 100 k$. We orient $F$ so that $\left|d^{-}(v)-d^{+}(v)\right| \leq 1$ for all $v \in$ LARGE. We now choose a subset $A_{v}$ of edges directed outward from $v$ by this orientation, of size $\left\lfloor(9 \log n) / 200 k^{2}\right\rfloor k$.

The following lemma, applied to the sets $A_{v}$ defined above, gives the required monochromatic set $E_{2}$.

Lemma 3 Let $A_{1}, A_{2}, \ldots, A_{n}$ be disjoint sets with $\left|A_{i}\right|=2 k-1,1 \leq i \leq r \leq k-1$ and $\left|A_{i}\right|=$

$m_{i} k, r+1 \leq i \leq n$, where the $m_{i}$ 's are positive integers. Let $A=A_{1} \cup A_{2} \cup \cdots \cup A_{n}$. Suppose that the elements of $A$ are coloured so that no colour is used more than $k$ times. Then there exists a multicoloured subset $B$ of $A$ such that $\left|A_{i} \cap B\right|=2,1 \leq i \leq r$ and $\left|A_{i} \cap B\right|=m_{i}, r+1 \leq i \leq n$. 
THE ELECTRONiC Journal of COMBinatorics 2 (1995), \#R19

Proof $\quad$ For $i=1, \ldots, r$ partition $A_{i}$ into $B_{i, 1}, B_{i, 2}$ where $\left|B_{i, 1}\right|=k-1$ and $\left|B_{i, 2}\right|=k$, and let $m_{i}=2$. For $i=r+1, \ldots, n$ partition $A_{i}$ into subsets $B_{i, j}\left(j=1, \ldots, m_{i}\right)$ of size $k$.

Let $X=\left\{B_{i, j}: i=1, \ldots, n, j=1, \ldots, m_{i}\right\}$ and let $Y$ be the set of colours used in the $k$-bounded colouring of $A$. We consider a bipartite graph $\Gamma$ with bipartition $(X, Y)$, where $(x, y)$ is an edge of $\Gamma$ if colour $y \in Y$ was used on the elements of $x \in X$.

We claim that $\Gamma$ contains an $X$-saturated matching. Let $S \subseteq X,|S|=s$, and suppose $t$ elements of $S$ are sets of size $k-1$ and $s-t$ are of size $k$. We have

$$
\begin{aligned}
\left|\bigcup_{B_{i, j} \in S} B_{i, j}\right| & =(s-t) k+t(k-1) \\
& =s k-t .
\end{aligned}
$$

Thus the set of neighbours $N_{\Gamma}(S)$ of $S$ in $\Gamma$ satisfies

$$
\left|N_{\Gamma}(S)\right| \geq\left\lceil s-\frac{t}{k}\right\rceil \geq\left\lceil s-\left(\frac{k-1}{k}\right)\right\rceil=|S|
$$

and $\Gamma$ satisfies Hall's condition for the existence of an $X$-saturated matching $M=\left\{\left(B_{i, j}, y_{i, j}\right)\right\}$. Now construct $B$ by taking an element of colour $y_{i, j}$ in $B_{i, j}$ for each $(i, j)$.

\subsection{Properties of $H=\left(V, E_{1} \cup E_{2}\right)$}

We first state or prove some basic properties of $H$.

Lemma $4 H$ is multicoloured, and $\delta(H) \geq 2$.

\section{Lemma 5 With high probability}

D3 $S \subseteq L A R G E,|S| \leq \frac{n}{100 \log n} \Longrightarrow\left|N_{H}(S)\right| \geq \frac{\epsilon \log n}{300 k^{2}}|S|$. 
THE ELECTRONiC Journal of COMBinatorics 2 (1995), \#R19

\section{Proof}

Case of $|S| \leq n /(\log n)^{3}$

If $S \subseteq$ LARGE, then $T=N_{H}(S) \cup S$ contains at least $\left\lfloor\frac{9}{200 k^{2}} \log n\right\rfloor|S| / 2$ edges in $E_{2}$. No subset $T$ of size at most $n /(\log n)^{2}$ contains more than $3|T|$ edges (by P5). Thus $|T| \geq\left\lfloor\frac{9}{200 k^{2}} \log n\right\rfloor|S| / 6$ and so

$$
\left|N_{H}(S)\right| \geq \frac{3}{500 k^{2}} \log n|S|
$$

Case of $n /(\log n)^{3}<|S| \leq n / 100 \log n$

By $\mathrm{P} 4, G$ satisfies $|N(S)| \geq(|S| \log n) / 20$ and we can choose a set $M$ of

$$
\lfloor(|S| \log n) / 20-(k \mid \text { SMALL } \mid \log n) / 10\rfloor
$$

edges which have one endpoint in $S$, the other a distinct endpoint not in $S$ and of a colour different to that of any edge incident with a vertex of SMALL. This set of edges contains at least $|M| / k$ colours. If $M$ contains $t$ edges of colour $i$ and $G$ contains $r$ edges of colour $i$ in total, then the probability $\rho$ that an edge of $M$ of colour $i$ is included in $E_{1}$ satisfies

$$
\rho \geq \frac{t \epsilon}{k}\left(1-\frac{\epsilon}{k}\right)^{r-1} \geq \frac{t \epsilon}{k}(1-\epsilon)>\frac{\epsilon}{2 k} .
$$

Thus $\left|N_{H}(S)\right|$ dominates $\operatorname{Bin}\left(\frac{|M|}{k}, \frac{\epsilon}{2 k}\right)$, and by (3)

$$
\operatorname{Pr}\left(\left|N_{H}(S)\right| \leq \frac{|M| \epsilon}{4 k^{2}}\right) \leq\left(\frac{2}{e}\right)^{|M| \epsilon / 4 k^{2}}
$$

Hence the probability that some set has less than the required number of neighbours to its neighbour set is

$$
\begin{aligned}
\sum_{s=n /(\log n)^{3}}^{n /(100 \log n)}\left(\begin{array}{l}
n \\
s
\end{array}\right)\left(\frac{2}{e}\right)^{(\epsilon s \log n) / 100 k^{2}} & \leq \sum_{s}\left[\exp -\left\{\frac{\epsilon \log (e / 2)}{100 k^{2}} \log n-4 \log \log n\right\}\right]^{s} \\
& =o(1) .
\end{aligned}
$$


THE ELECTRONIC JOURNAL OF COMBINATORICS 2 (1995), \#R19

Lemma 6 Let $D \geq \frac{32 k^{2}}{\epsilon}$; if $|A|,|B| \geq D n \frac{\log \log n}{\log n}$ then whp

D4 $H$ contains more than $\left\lfloor\frac{2}{D}|A||B| \frac{\log n}{n}\right\rfloor$ edges between $A$ and $B$.

Proof The proof follows that of Lemma 5. By P6, the number of edges between $A$ and $B$ in $G$ of a colour different to that of any edge incident with a vertex of SMALL is at least $M=\lfloor(|A||B| \log n / 2 n)-(k \mid$ SMALL $\mid \log n) / 10\rfloor$. Thus the number of $E_{1}$-edges between these sets dominates $\operatorname{Bin}(M / k, \epsilon / 2 k)$. Let $K=(1-o(1)) \frac{8(1-(\log 4 e) / 4)}{D} \frac{\log n}{n}$. The probability that there exist sets $A, B$ with at most $\left\lfloor\frac{2}{D}|A||B| \frac{\log n}{n}\right\rfloor E_{1}$-edges between them is (by (3)) at most

$$
\begin{aligned}
\sum_{a, b}\left(\begin{array}{l}
n \\
a
\end{array}\right)\left(\begin{array}{l}
n \\
b
\end{array}\right)\left(\frac{(4 e)^{\frac{1}{4}}}{e}\right)^{(1-o(1)) \frac{M \epsilon}{2 k^{2}}} & \leq \sum_{a, b}\left(\frac{n e}{a}\right)^{a}\left(\frac{n e}{b}\right)^{b} e^{-K a b} \\
& \leq \sum_{a, b} \exp \{(a+b) \log \log n-K a b\} \\
& \leq \sum_{a, b} \exp \left\{a b\left(\left(\frac{1}{a}+\frac{1}{b}\right) \log \log n-K\right)\right\} \\
& \leq \sum_{a, b} \exp \left\{a b\left(\frac{2 \log n}{D n}-\frac{3 \log n}{D n}\right)\right\} \\
& \leq n^{2} \exp \left\{-D n \frac{(\log \log n)^{2}}{\log n}\right\} \\
& =o(1) .
\end{aligned}
$$

Assume from now on that $H$ satisfies D1-D4. We note the following immediate Corollary.

Corollary 1 whp $H$ is connected. 
THE ELECTRONiC JOURNAL OF COMBinAtorics 2 (1995), \#R19

Proof If $H$ is not connected then from D4 its has a component $C$ of size at $\operatorname{most} D n \frac{\log \log n}{\log n}$. But then D3 and P3 imply $C \cap \mathrm{LARGE}=\emptyset$. Now apply D1 and P2 to get a contradiction.

\section{Proof that $H$ is Hamiltonian}

Let us suppose we have selected a $G$ satisfying properties $\mathrm{P} 1-\mathrm{P} 10$, and sampled a suitable $H$ which satisfies D1-D4. We now show that it must follow that $H$ contains a multicoloured Hamilton cycle.

\subsection{Construction of an initial long path}

We use rotations and extensions in $H$ to find a maximal path with large rotation endpoint sets, see for example [6], [14]. Let $P_{0}=\left(v_{1}, v_{2}, \ldots, v_{l}\right)$ be a path of maximum length in $H$. If $1 \leq i<l$ and $\left\{v_{l}, v_{i}\right\}$ is an edge of $H$ then $P^{\prime}=\left(v_{1} v_{2} \ldots v_{i} v_{l} v_{l-1} \ldots v_{i+1}\right)$ is also of maximum length. It is called a rotation of $P_{0}$ with fixed endpoint $v_{1}$ and pivot $v_{i}$. Edge $\left(v_{i}, v_{i+1}\right)$ is called the broken edge of the rotation. We can then, in general, rotate $P^{\prime}$ to get more maximum length paths.

Let $S_{t}=\left\{v \in\right.$ LARGE : $v \neq v_{1}$, is the endpoint of a path obtainable from $P_{0}$ by $t$ rotations with fixed endpoint $v_{1}$ and all broken edges in $\left.P_{0}\right\}$.

It follows from P3 and D3 that $S_{1} \neq \emptyset$. It then follows that if $\left|S_{t}\right| \leq n /(100 \log n)$ then $\left|S_{t+1}\right| \geq$ $\epsilon \log n\left|S_{t}\right| /\left(1000 k^{2}\right)$, for making this inductive assumption which is true for $\left|S_{1}\right|$ by D2,

$$
\begin{aligned}
\left|S_{t+1}\right| & \geq\left|N_{H}\left(S_{t}\right)\right| / 2-\left(1+\left|S_{1}\right|+\left|S_{2}\right|+\cdots\left|S_{t}\right|\right) \\
& \geq \epsilon \log n\left|S_{t}\right| /\left(600 k^{2}\right)-\left(1+\left|S_{1}\right|+\left|S_{2}\right|+\cdots\left|S_{t}\right|\right) \\
& \geq \epsilon \log n\left|S_{t}\right| /\left(1000 k^{2}\right) .
\end{aligned}
$$

Thus there exists $t_{0} \leq(1+o(1)) \log n / \log \log n$ such that $\left|S_{t_{0}}\right| \geq c n, c=\epsilon /\left(10^{6} k^{2}\right)$. Let $B\left(v_{1}\right)=$ $S_{t_{0}}$ and $A_{0}=B\left(v_{1}\right) \cup\left\{v_{1}\right\}$. Similarly, for each $v \in B\left(v_{1}\right)$ we can construct a set of endpoints 
THE ELECTRONiC JOURNAL OF COMBinAtorics 2 (1995), \#R19

$B(v),|B(v)| \geq c n$ of endpoints of maximum length paths with endpoint $v$. Note that $l \geq c n$ as every vertex of $B_{0}$ lies on $P_{0}$.

In summary, for each $a \in A_{0}, b \in B(a)$ there is a maximum length path $P(a, b)$ joining $a$ and $b$ and this path is obtainable from $P_{0}$ by at most $(2+o(1)) \log n / \log \log n$ rotations.

\subsection{Closure of the maximal path}

This section follows closely both the notation and the proof methodology used in [1].

Given path $P_{0}$ and a set of vertices $S$ of $P_{0}$, we say $s \in S$ is an interior point of $S$ if both neighbours of $s$ on $P_{0}$ are also in $S$. The set of all interior points of $S$ will be denoted by $\operatorname{int}(S)$.

Lemma 7 Given a set $S$ of vertices with $|\operatorname{int}(S)| \geq 7 D n \frac{\log \log n}{\log n}, D \geq 32 k^{2} / \epsilon$ there is a subset $S^{\prime} \subseteq S$ such that, for all $s^{\prime} \in S^{\prime}$ there are at least $m=\frac{1}{D} \frac{\log n}{n}|\operatorname{int}(S)|$ edges between $s^{\prime}$ and $\operatorname{int}\left(S^{\prime}\right)$. Moreover, $\left|\operatorname{int}\left(S^{\prime}\right)\right| \geq|\operatorname{int}(S)| / 2$.

Proof We use the proof given in [1]. If there is a $s_{1} \in S$ such that the number of edges from $s_{1}$ to $\operatorname{int}(S)$ is less than $m$ we delete $s_{1}$, and define $S_{1}=S \backslash\left\{s_{1}\right\}$. If possible we repeat this procedure for $S_{1}$, to define $S_{2}=S_{1} \backslash\left\{s_{2}\right\}$ (etc). If this continued for $r=\left\lfloor\frac{1}{6}|\operatorname{int}(S)|\right\rfloor$ steps, we would have a set $S_{r}$ and a set $R=\left\{s_{1}, s_{2}, \ldots, s_{r}\right\}$, with

$$
\left|\operatorname{int}\left(S_{r}\right)\right| \geq|\operatorname{int}(S)|-3|R| \geq|\operatorname{int}(S)|-3 r \geq \frac{|\operatorname{int}(S)|}{2} .
$$

This step follows because deleting a vertex of $S$ removes at most 3 vertices of int $(S)$. However, there are fewer than

$$
\begin{aligned}
m|S| & \leq \frac{1}{D} \frac{\log n}{n}|\operatorname{int}(S)||R| \\
& \leq \frac{2}{D} \frac{\log n}{n}\left|\operatorname{int}\left(S_{r}\right)\right||R|
\end{aligned}
$$


edges from $R$ to $\operatorname{int}\left(S_{r}\right)$, which contradicts our assumption D4.

In Section 7.1 we proved the existence of maximum length paths $P(a, b), b \in B(a), a \in A_{0}$ where $\left|A_{0}\right|,|B(a)| \geq c n$. Thus there are at least $c^{2} n^{2}$ distinct endpoint pairs $(a, b)$ and for each such pair there is a path $P(a, b)$ derived from at most $\rho=(2+o(1)) \log n / \log \log n$ rotations starting with some fixed maximal path $P_{0}$.

We consider $P_{0}$ to be directed and divided into $2 \rho$ segments $I_{1}, I_{2}, \ldots, I_{2 \rho}$ of length at least $\left\lfloor\left|P_{0}\right| / 2 \rho\right\rfloor$, where $\left|P_{0}\right| \geq c n$. As each $P(a, b)$ is obtained from $P_{0}$ by at most $\rho$ rotations, the number of segments of $P_{0}$ which occur on this path, although perhaps reversed, is at least $\rho$. We say that such a segment is unbroken. These segments have an absolute orientation given by $P_{0}$, and another, relative to this by $P(a, b)$, which we regard as directed from $a$ to $b$. Let $t$ be a fixed natural number. We consider sequences $\sigma=I_{i_{1}}, \ldots, I_{i_{t}}$ of unbroken segments of $P_{0}$, which occur in this order on $P(a, b)$, where we consider that $\sigma$ also specifies the relative orientation of each segment. We call such a sequence $\sigma$ a $t$-sequence, and say $P(a, b)$ contains $\sigma$.

For given $\sigma$, we consider the set $L=L(\sigma)$ of ordered pairs $(a, b), a \in A_{0}, b \in B(a)$ which contain the sequence $\sigma$.

The total number of such sequences of length $t$ is $(2 \rho)_{t} 2^{t}$. Any path $P(a, b)$ contains at least $\rho \geq \log n / \log \log n$ unbroken segments, and thus at least $\left(\begin{array}{l}\rho \\ t\end{array}\right) t$-sequences. The average, over $t$ sequences, of the number of pairs containing a given $t$-sequence is therefore at least

$$
\frac{c^{2} n^{2}\left(\begin{array}{l}
\rho \\
t
\end{array}\right)}{(2 \rho)_{t} 2^{t}} \geq \alpha n^{2}
$$

where $\alpha=c^{2} /(4 t)^{t}$. Thus there is a $t$-sequence $\sigma_{0}$ and a set $L=L\left(\sigma_{0}\right),|L| \geq \alpha n^{2}$ of pairs $(a, b)$ such that for each $(a, b) \in L$ the path $P(a, b)$ contains $\sigma_{0}$. Let $\hat{A}=\{a: L$ contains at least $\alpha n / 2$ pairs with $a$ as first element $\}$. Then $|\hat{A}| \geq \alpha n / 2$. For each $a \in \hat{A}$ let $\hat{B}(a)=\{b:(a, b) \in L\}$.

Let $t=1700 D^{2} / c, D=32 k^{2} / \epsilon$ and let $C_{1}$ denote the union of the first $t / 2$ segments of $\sigma_{0}$, in the fixed order and with the fixed relative orientation in which they occur along any of the paths 
$P(a, b),(a, b) \in L$. Let $C_{2}$ denote the union of the second $t / 2$ segments of $\sigma_{0} . C_{1}$ and $C_{2}$ contain at least $\frac{t}{2} c n \frac{\log \log n}{4 \log n}(1-o(1))$ interior points which from Lemma 7 gives sets $C_{1}^{\prime}, C_{2}^{\prime}$ with at least

$$
\frac{t c(1-o(1))}{16} n \frac{\log \log n}{\log n} \geq 100 D^{2} n \frac{\log \log n}{\log n}
$$

interior points.

It follows from D4 that there exists $\hat{a} \in \hat{A}$ such that $H$ contains an edge from $\hat{a}$ to $C_{1}^{\prime}$. Similarly, $H$ contains an edge joining some $\hat{b} \in \hat{B}(\hat{a})$ to $C_{2}^{\prime}$. Let $x$ be some vertex separating $C_{1}^{\prime}$ and $C_{2}^{\prime}$ along $\hat{P}=P(\hat{a}, \hat{b})$. We now consider the two half paths $P_{1}, P_{2}$ obtained by splitting $\hat{P}$ at $x$. We consider rotations of $P_{i}, i=1,2$ with $x$ as a fixed endpoint. We show that in both cases the finally constructed endpoint sets $V_{1}, V_{2}$ are large enough so that D4 guarantees an edge from $V_{1}$ to $V_{2}$. We deduce that $H$ is Hamiltonian as the path it closes is of maximum length and $H$ is connected.

Consider $P_{1}$. Let $T_{i}=\left\{v \in C_{1}^{\prime}: v \neq x\right.$ is the endpoint of a path obtainable from $P_{1}$ by $t$ rotations with fixed endpoint $x$, pivot in $\operatorname{int}\left(C_{1}^{\prime}\right)$ and all broken edges in $\left.P_{1}\right\}$. We claim we can choose sets $U_{i} \subseteq T_{i}, i=1,2, \ldots$ such that $\left|U_{1}\right|=1$ and $\left|U_{i+1}\right|=2\left|U_{i}\right|$, as long as $\left|U_{i}\right| \leq D n \frac{\log \log n}{\log n}$. Thus there is an $i^{*}$ such that $\left|U_{i^{*}}\right| \geq D n \frac{\log \log n}{\log n}$ and we are done. Note that $T_{1} \neq \emptyset$ because $\hat{a}$ has an $H$-neighbour in $\operatorname{int}\left(C_{1}^{\prime}\right)$. Note also that if we make a rotation with pivot in $\operatorname{int}\left(C_{1}^{\prime}\right)$ and broken edge in $P_{1}$ then the new endpoint created is $C_{1}^{\prime}$.

Let $y$ be a vertex of $U_{i}$. Then by Lemma 7 there are at least $100 D \log \log n$ edges between $y$ and $\operatorname{int}\left(C_{1}^{\prime}\right)$. Thus the number of edges from $U_{i}$ to $\operatorname{int}\left(C_{1}^{\prime}\right)$ is at least $50\left|U_{i}\right| D \log \log n$. As $\left|\bigcup_{j=1}^{i} U_{i}\right|<$ $2\left|U_{i}\right|$ at most $20 D\left|U_{i}\right| \log \log n$ of these edges are contained in $\bigcup_{j=1}^{i} U_{j}$ (from P8), and so by $\mathrm{P} 7$ we have $\left|T_{i+1}\right|>2\left|U_{i}\right|$ and we select a subset of size exactly $2\left|U_{i}\right|$. 
THE ELECTRONIC JOURNAL OF COMBINATORICS 2 (1995), \#R19

\section{Similar Problems}

We note that it is straightforward to extend the above analysis to find the corresponding thresholds when Hamilton cycle is replaced by perfect matching or spanning tree. Now whp one needs enough edges so that the following replacements for conditions P9 and P10 hold true.

P9a $G$ has minimum degree $k-1$.

P10a $G$ has at most $k-1$ vertices of degree $k-1$

That these conditions are necessary can be argued as in Section 4, since connectivity and the existence of a perfect matching require minimum degree one. Lemma 3 is replaced by

Lemma 8 Let $A_{1}, A_{2}, \ldots, A_{n}$ be disjoint sets with $\left|A_{i}\right|=k-1,1 \leq i \leq r \leq k-1$ and $\left|A_{i}\right|=$ $m_{i} k, r+1 \leq i \leq n$, where the $m_{i}$ 's are positive integers. Let $A=A_{1} \cup A_{2} \cup \cdots \cup A_{n}$. Suppose that the elements of $A$ are coloured so that no colour is used more than $k$ times. Then there exists a multicoloured subset $B$ of $A$ such that $\left|A_{i} \cap B\right|=1,1 \leq i \leq r$ and $\left|A_{i} \cap B\right|=m_{i}, r+1 \leq i \leq n$.

The proof is the same.

We choose $E_{1}$ and $E_{2}$ in the same way as before. The fact that $H$ is connected proves the existence of a multicoloured tree. For a perfect matching one can remove from $H$ all vertices of degree one together with their neighbours and argue that the graph that remains is Hamiltonian (assuming $n$ is even). The proof is essentially that of Section 7 . 
THE ELECTRONiC JOURNAL OF COMBinAtorics 2 (1995), \#R19

\section{References}

[1] M. Ajtai, J. Komlós and E. Szemerédi. The first occurrence of Hamilton cycles in random graphs. Annals of Discrete Mathematics 27 (1985) 173-178.

[2] M.J. Albert, A.M. Frieze and B. Reed, Multicoloured Hamilton Cycles. Electronic Journal of Combinatorics 2 (1995) R10.

[3] B. Bollobás. The evolution of sparse graphs. Graph Theory and Combinatorics. (Proc. Cambridge Combinatorics Conference in Honour of Paul Erdős (B. Bollobás; Ed)) Academic Press (1984) $35-57$

[4] B. Bollobás. Random Graphs. Academic Press (1985)

[5] B. Bollobás and A.M. Frieze, On matchings and Hamiltonian cycles in random graphs. Annals of Discrete Mathematics 28 (1985) 23-46.

[6] B. Bollobás, T.I. Fenner and A.M. Frieze. An algorithm for finding Hamilton cycles in random graphs. Combinatorica 7 (1987) 327-341.

[7] B. Bollobás, T. Fenner and A.M. Frieze. Hamilton cycles in random graphs with minimal degree at least k. (A Tribute to Paul Erdős (A.Baker, B.Bollobas and A.Hajnal; Ed)) (1990) 59-96.

[8] C. Cooper. 1-pancyclic Hamilton cycles in random graphs. Random Structures and Algorithms 3.3 (1992) 277-287

[9] C. Cooper and A. Frieze. Pancyclic random graphs. Proc. 3rd Annual Conference on Random Graphs, Poznan 1987. Wiley (1990) 29-39

[10] C. Cooper and A. Frieze. On the lower bound for the number of Hamilton cycles in a random graph. Journal of Graph Theory 13.6 (1989) 719-735 
THE ELECTRONIC JOURNAL OF COMBINATORICs 2 (1995), \#R19

[11] P. Erdős and A. Rényi. On the strength of connectedness of a random graph. Acta. Math. Acad. Sci. Hungar. 12 (1961) 261-267.

[12] A. Frieze and B. Reed. Polychromatic Hamilton cycles. Discrete Maths. 118 (1993) 69-74.

[13] G. Hahn and C. Thomassen. Path and cycle sub-Ramsey numbers, and an edge colouring conjecture. Discrete Maths. 62 (1986) 29-33

[14] J. Komlós and E. Szemerédi. Limit distributions for the existence of Hamilton cycles in a random graph. Discrete Maths. 43 (1983) 55-63.

[15] T. Łuczak. Cycles in random graphs. Discrete Maths. (1987)

[16] T. Euczak, On the equivalence of two basic models of random graph, Proceedings of Random graphs 87, Wiley, Chichester (1990), 151-159.

[17] Rödl and A. Ruciński, Threshold functions for Ramsey properties. (to appear)

[18] Rödl and Winkler. Private communication (1984) 
THE ELECTRONiC JOURNAL OF COMBinAtorics 2 (1995), \#R19

\section{Appendix: Proofs of P6-P8}

P5 $T \subseteq V,|T| \leq n /(\log n)^{2}$ implies $T$ contains at most $3|T|$ edges.

The number of edges in $T$ is $\operatorname{Bin}\left(\left(\begin{array}{c}|T| \\ 2\end{array}\right), p\right)$. By (4) the probability that there exists $T$ with $3|T|$ edges is at most

$$
\begin{aligned}
\sum_{t=7}^{n /(\log n)^{2}}\left(\begin{array}{l}
n \\
t
\end{array}\right)\left(\frac{e\left(\begin{array}{l}
t \\
2
\end{array}\right) p}{3 t}\right)^{3 t} e^{-\left(\begin{array}{l}
t \\
2
\end{array}\right) p} & \leq \sum_{t}\left(\frac{n e}{t}\right)^{t}\left(\frac{(1+o(1)) e t \log n}{6 n}\right)^{3 t} \\
& \leq \sum_{t}\left(\frac{(\log n)^{3} t^{2}}{n^{2}}\right)^{t} \\
& =o(1) .
\end{aligned}
$$

P6 $A, B \subseteq V, A \cap B=\emptyset,|A|,|B| \geq 15 n \log \log n / \log n$ implies $G$ contains at least $|A||B| \log n / 2 n$ edges joining $A$ and $B$.

The number of edges between $A$ and $B$ is $\operatorname{Bin}(|A||B|, p)$. By (3), the probability there exist sets $A, B$ with less than half the expected number of edges between them, is at most

$$
\begin{aligned}
\sum_{a, b}\left(\begin{array}{l}
n \\
a
\end{array}\right)\left(\begin{array}{l}
n \\
b
\end{array}\right)\left(\frac{2}{e}\right)^{a b p} & \leq \exp \{a \log (n e / a)+b \log (n e / b)-a b p \log (e / 2)\} \\
& \leq n^{2} \exp \left\{-\frac{n(\log \log n)^{2}}{\log n}(15)^{2}(\log (e / 2)-2 / 15)\right\} \\
& =o(1),
\end{aligned}
$$

by the same arguments as those following (6) in Lemma 6.

P7 $A, B \subseteq V, A \cap B=\emptyset,|A| \leq|B| \leq 2|A|$ and $|B| \leq D n \log \log n / \log n(D \geq 1)$ implies that there are at most $10 D|A| \log \log n$ edges joining $A$ and $B$.

Let $|B|=2|A|=2 a$. We have then that the probability that there exist $A, B$ such that there are 
at least $10 D|A| \log \log n$ edges between the sets is at most

$$
\begin{aligned}
\sum_{a}\left(\begin{array}{l}
n \\
a
\end{array}\right)\left(\begin{array}{c}
n \\
2 a
\end{array}\right)\left(\begin{array}{c}
2 a^{2} \\
10 D a \log \log n
\end{array}\right) p^{10 D a \log \log n} & \leq \sum_{a}\left[\left(\frac{n e}{a}\right)\left(\frac{n e}{2 a}\right)^{2}\left(\frac{a e \log n}{5 D n \log \log n}\right)^{10 D \log \log n}\right]^{a} \\
& \leq \sum_{a}\left[\frac{e^{3}}{4}\left(\frac{\log n}{2 D \log \log n}\right)^{3}\left(\frac{e}{5}\right)^{10 D \log \log n}\right]^{a} \\
& \leq \sum_{a}\left(\frac{1}{\log n}\right)^{a} \\
& =o(1) .
\end{aligned}
$$

P8 If $|A| \leq D n \log \log n / \log n(D \geq 1)$ then $A$ contains at most $10 D|A| \log \log n$ edges.

We may assume $|A|>n /(\log n)^{2}$ by P5. The number of induced edges in $A$ is $\operatorname{Bin}\left(\left(\begin{array}{c}|A| \\ 2\end{array}\right), p\right)$. By (4) the probability there exists a set $A$ with at least $20(1-o(1))$ times the expected number of edges is at most,

$$
\begin{aligned}
\sum_{a}\left(\begin{array}{l}
n \\
a
\end{array}\right)\left(\frac{e}{19}\right)^{10 D a \log \log n} & \leq \sum_{a} n \exp \{a \log (n e / a)-10 D a \log \log n \log (19 / e)\} \\
& \leq \sum_{a} n \exp \left\{-\frac{D n \log \log n}{(\log n)^{2}}(10 \log (19 / e)-2)\right\} \\
& =o(1) .
\end{aligned}
$$

